Oladeji and Agbelusi, Afr J Tradit Complement Altern Med., (2017) 15 (1): 117-136

https://doi.org/10.21010/ajtcam.v15i1.12

\title{
CAPTURING INDIGENOUS KNOWLEDGE ON MEDICINAL PLANTS USE: CASE STUDY OF SELECTED COMMUNITIES IN OLD OYO NATIONAL PARK, NIGERIA
}

\section{S. O. Oladeji * and E.A Agbelusi}

Department of Ecotourism and Wildlife Management, Federal University of Technology, Akure.

*Corresponding Author Email: sooladeji@ fua.edu.ng, oladejisunny@yahoo.com

\begin{tabular}{|c|}
\hline \multicolumn{1}{|c|}{ Article History } \\
Received: May. 10, 2017 \\
Revised Received: Aug. 29, 2017 \\
Accepted: Aug. 31, 2017 \\
Published Online: Dec. 29, 2017 \\
\hline
\end{tabular}

\begin{abstract}
Background: Understanding the intricate link between humans and the plants use is needed to address some of to-day's socio-economic and health challenges.

Materials and Methods: The study focused on plants that have high cultural and medicinal values for the host communities of Old Oyo National Park, Nigeria. Local Knowledge was captured through focus group discussions with herbal vendors, interviews with herbalists as key informants and through field observations. Snowball sampling technique was used in selecting the key informants. Participants selected for the Focus Group Discussion varies with the number of herbal vendors encountered in each commercial market.

Results: In total, 78 medicinal plant species belonging to 39 families including 47 trees, 8 shrubs and 23 herbaceous plants were documented. Forty ailments categorized into thirty one treatments and prevention methods were discovered. Kigellia africana, Cassia spp and Alstonia boonei were used for treating common ailments like malaria, dysentery and gonorrhea. Sources of collecting the medicinal plant species and their mode of administration were different.

Conclusion: The research outcome will contribute to advancement of traditional medicine, resource efficiency and development of new enterprise among the locals. Comprehensive quantitative study of social- economic importance of the medicinal plants is recommended.
\end{abstract}

Keywords: Intricate link, cultural values, host communities, traditional medicine, resource efficiency.

\section{Introduction}

There is a close cultural link between people and plants particularly in terms of treatment for ailments and nutrition. There is also an interesting similarity between the socio-economic and cultural values of the two subjects. Many of the planet's areas of highest biological diversity are inhabited by indigenous people with strong traditional ties (Owuor and Kisangau, 2006) providing what the Declaration of Belem called an "inextricable link" between biological and cultural diversity (Posey 1999). To describe the concept denoting this link, the term biocultural diversity was introduced (Posey 1988). Indigenous knowledge of medicinal plant use is context specific as it is related to, and contained within, a group of people who live in a defined geographic region (Kassam et al. 2010). Knowledge in this context is derived from a web of interactions among humans, plants, animals, natural forces, and land forms.

The use of traditional medicine and medicinal plants in most developing countries has been widely observed (UNESCO 1996). It was estimated that nearly three fourth of the plant -derived prescription drugs used worldwide were discovered following leads from local medicine (Wakdikar 2004). Development of modern biotechnological techniques therefore depends on the information obtained from the traditional healers; since they are found in most local communities deeply entrenched in their culture, tradition and belief. Hence, integration of traditional healers into primary health care has been emphasized in the literatures (Owuor et al 2005; Owuor and Kisangu 2006) as an approach to achieve improvement in health care system. 
Traditional and folklore medicine handed down from generation to generation is rich in household remedies and community practice (Subramanyam et al. 2008). Traditional healers are found at household levels and they possess in-depth understanding of the medicinal plants used for treating various forms of ailments. Medicinal plants are used at the household level and over $80 \%$ of the populations of developing countries rely on traditional medicine, mostly plant drug, for their primary health care needs (WHO 1980). Traditional healers are often the first and last line of defense against most diseases such as headaches, coughs, diarrhea, wound healing and skin diseases (Matsheta and Mulaudzi 2008).

Since the beginning of this century, there has been an increasing interest in the study of medicinal plants and their traditional use in different parts of the world (Uprety et al. 2010). Recent interest in this area of research is partly driven by society's interest in healthy lifestyles, which supports a rapidly growing $\$ 230$ billion dollar market force in USA alone (Pesek et al. 2006) About 25\% of modern medicines are descended from plants first used traditionally. Many others are synthetic analogues built on prototype compounds isolated from plants. About $70 \%$ modern medicines in India for example, are derived from natural products (Choudhary 2002). It is estimated that there are over 7800 medicinal drug manufacturing units in India, which consume about 2000 tonnes of herbs annually (Ramakrishnappa 2002).

The increasing global demand for herbal medicine has aroused global interest in ethno-botanical studies in recent times (Srivasta 2000). Medicinal plant uses contribute to the establishment of industries, employment generation and increased income ( Baishya and Begum 2013). For instance in Costa- Rica substantial amount of money is being generated from the sales of medicinal plants and derived products (Ceballous-Lascurain 2001).

Despite the long standing history and culture of the host communities of Old Oyo National Park as recorded in some of the literatures (Akinjogbin 1996; Agbaje-Williams 1983 and Alarape 2002), the knowledge of the local people in medicinal plant use has not been properly documented. The site of Oyo-Ile in the north east corner of the Park was used as capital of Oyo-Empire between the $17^{\text {th }}$ and $18^{\text {th }}$ centuries (Akinjogbin 1966). The researcher emphasized that this was the hub of the ancient Oyo empire, a historic city and one of the first states to emerge in the forest and the coastal region of West Africa. The empire was flourished in the $18^{\text {th }}-19^{\text {th }}$ centuries as an important urban center both economically and politically; the present Oyo might be the seventh or eighth capitals (Agbaje-Williams 1983). This was among the key factors for the initiation of this research work hinged on the need to provide useful and detail information on the medicinal values of the flora resources based on the local knowledge of the host communities of Old Oyo National Park.

The study captured indigenous knowledge of medicinal plants used in selected communities adjoining the Park. Methods of use and preparation, ailments being treated, plant use frequency, distribution and extraction methods were documented. The research findings will contribute to advancement of contemporary medicine through the development of new enterprise and increase natural resource use. This will facilitate development of benefit sharing scheme targeting the alleviation of poverty and improving the livelihood of the local communities.

\section{Methods and Materials Study area}

This study was conducted in Old Oyo National Park, Oyo-State, Nigeria. Politically, the park lies in Oyo State in the Southwest of Nigeria and borders with Kwara State in the Northeast. The Park is located between $8^{\circ} 10^{\prime \prime}$ and $9^{\circ} 05^{\prime \prime}$ North latitudes, and $3^{\circ} 35^{\prime \prime}$ and $4^{\circ} 21^{\prime \prime}$ East longitudes (Figure 1). It covers a land area of approximately 2,512 square kilometers making it the fourth largest national park in Nigeria. The park spans through eleven Local Government Areas of Oyo state Atisbo (Tede/Ago-Are) $\left.3.4220^{\circ} \mathrm{E}, 8.5420^{\circ} \mathrm{N}\right)$, Atiba $(\mathrm{Oyo})\left(3.9260^{\circ} \mathrm{E}, 7.8400^{\circ} \mathrm{N}\right)$, Irepo $(\mathrm{Kisi})\left(3.8510^{\circ} \mathrm{E}, 9.079^{0} \mathrm{~N}\right)$, Oorelope (Igboho) $\left(3.7550^{\circ} \mathrm{E}, \quad 8.8340^{\circ} \mathrm{N}\right)$, Saki East (Ago-Amodu) $\left(3.6100^{\circ} \mathrm{E}, \quad 8.6090^{\circ} \mathrm{N}\right)$, Iseyin (Iseyin) $\left(3.5760^{\circ} \mathrm{E}, 7.9590^{\circ} \mathrm{N}\right)$, Orire (Ikoyi) $\left(4.1690^{\circ} \mathrm{E}, \quad 8.2700^{\circ} \mathrm{N}\right)$ Itesiwaju (Otu) $\left(3.3970^{0} \mathrm{E}, 8.2110^{\circ} \mathrm{N}\right), \quad$ Oyo West (Ojongbodu/Oyo), Olorunsogo (Igbeti( $\left.4.1350^{\circ} \mathrm{E}, 8.7450^{\circ} \mathrm{N}\right)$, Saki West (Saki) $\left(3.3860^{0} \mathrm{E}, 8.6620^{\circ} \mathrm{N}\right)$ and Kaima Local Government Area in Kwara State. The host communities adjoining the Park are selected for this study based on the long standing history of their existence and cultural antecedent as reported in some literatures (Akinjogbin, I.A 1996,; AgbajeWilliams, B 1983 and Alarape, A., 2002). The natural vegetation of the area is classified as Southern Guinea Savanna (Keay, 1959). However more intense studies by Charter (1970) classified the Southern portion of the vegetation as Forest savanna Mosaic with wooded savanna containing relic of Moist Semi deciduous forest, grading northwards into drier Mixed Leguminous Wooded Savanna with a continuous lower stratum of perennial grasses. Geerling (1973) analyzed and classified the vegetation into four broad groups; including dense woodland and forest outlier in the Southern portion and the North West corner dominated by Burkea africana, Afzelia africana, Detarium microcarpum,Daniellia oliveri, Pterocarpus erinaceus, Piliostigma thonningii, Vitex doniana, Nauclea latifolia. Mixed open savanna in the middle and north east portions dominated by Terminalia aviceniodes, Prosopis africana, Terminalia laxiflora and Khaya senegalensis. Outcrop vegetation in the hilly and rocky areas throughout, dominated by Adasonia digitata, Anogeisus leocarpus, Parkia biglobosa and Acacia species; Riparian grassland and fringing woodland and forest vary along major rivers and streams. The research work carried out by Afolayan, et al. (1996 \& 1997) gave a classification of the Vegetation of the Park to four broad ecozones. Zone $\mathrm{A}$ is designated as forest and dense savanna mosaic woodland of the park around Sepeteri axis with predominant species include Burkea africana, Afzelia africana, Detarium microcarpum, Piliostigma thonningii, Daniellia 
oliveri, Vitex doniana, Nauclea latifolia, Terminalia glaucescens, Kigelia africana, Anogeissus leiocarpus and Diosporous soubrena; Zone B as dense and open savanna woodland mosaic in the central portion of the park, Igbeti-Ighoho axis, dominated by species such as Terminalia aviceniodes, Prosopis africana, Terminalia laxiflora and Khaya senegalensis; Zone C as dense savanna woodland, north of Igbeti-Kishi axis and the dominant species include Burkea africana, Detarium microcarpum, Gardenia tenuifolia, Crossopteryx febrifuga, Ficus ingene, Ammania auriculata; and Zone D as open savanna woodland, North-east of the park (Oyo-Ile sector) with major tree species including Urena lobata, Pittosporum viridiflorum, Terminalia laxiflora, Ammania aurculata, Panicum repens, Parkia biglobosa, Cassia rotundifolia, Cleome ciliata. Findings from these studies have indicated changes in vegetation vis -a-vis species composition of the park. Over the years, the family Caesalpinioideae constituted the highest in species composition followed by the family Combretacea. Predominant floral resources in the Park include Burkea africana, Afzelia africana, Detarium microcarpum, Piliostigma thonningii, Daniellia oliveri, Vitex doniana, Nauclea latifolia, Terminalia glaucescens, Kigelia africana, Anogeissus leiocarpus and Diosporous soubrena. However, species of plants such as Vitex doniana, Diosporous soubrena ,Nauclea latifolia, Terminalia species have shown decrease tendency.

The flora resources experienced a decrease as a result of human activities such as collection for charcoal production, one of the major occupations of the inhabitants and collection for medicinal purposes (Yakubu and Idumah 2002). The park for a very long period in the past evidently suffered from mismanagement through destructive activities such as hunting, cattle grazing, logging and uncontrolled burning. The flora and fauna resources of the park have been largely depleted leading to local extermination of some species (Alarape 2002; Oladeji and Agbelusi 2014).

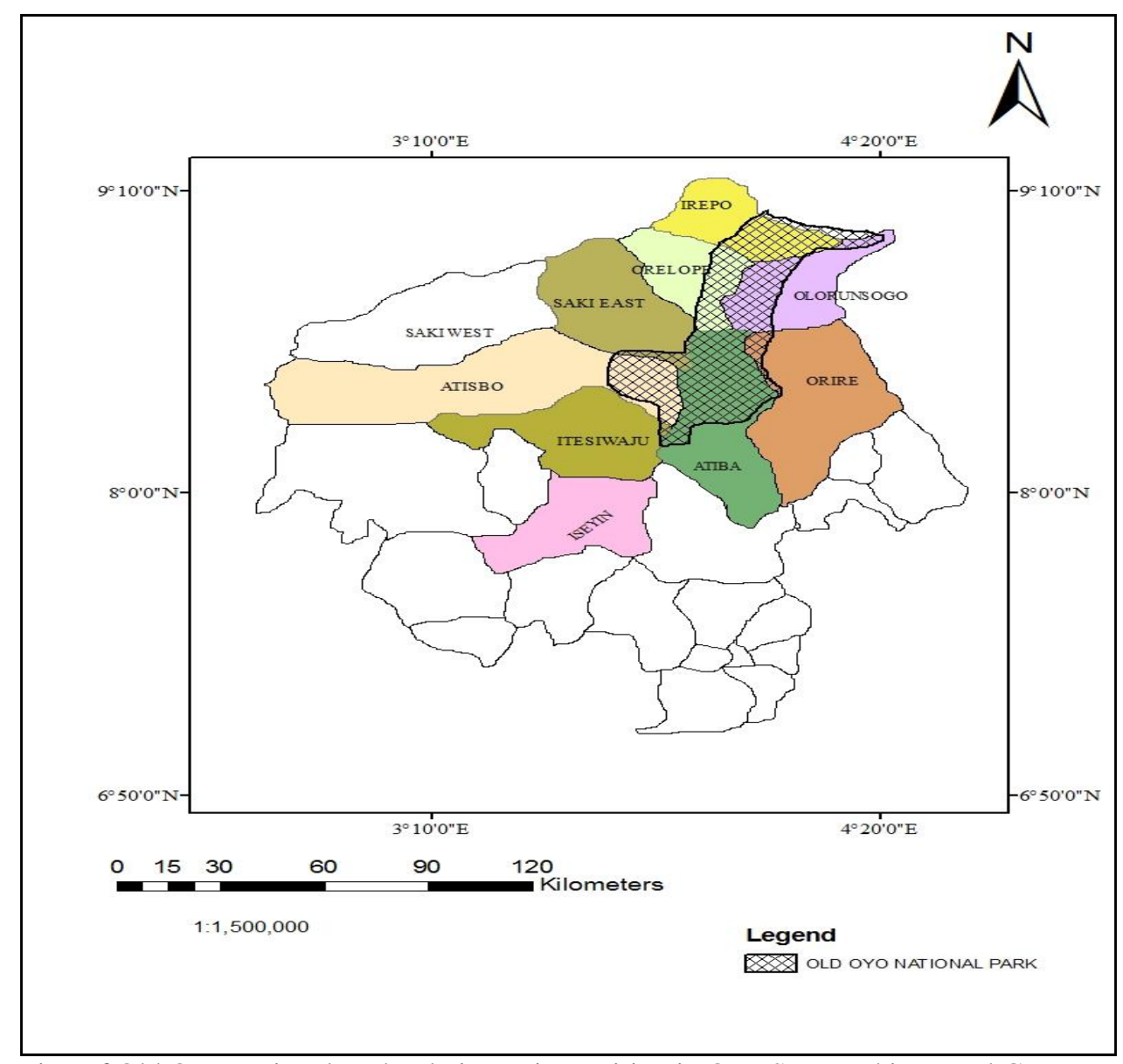

Figure 1: Location of Old Oyo National Park relative to its position in Oyo-State and its Local Government Areas. The study focused on four Local Government Areas: Iseyin, Atiba, Saki East and Atisbo.

\section{Method of data collection}

Focus Group Discussion, Key Informant Interview and field observations were employed for this study (Anwana and Obot 2003; Betti., 2004 and Kayode., 2006 ). For this study, four Local Government Areas (LGAs) were specifically selected from the ten LGAs adjacent to the Park, based on their proximities. The selected LGAs were Iseyin, Atiba, Saki East and Atisbo. Prior to the commencement of the research work, brief group discussions were held with the herbal 
vendors, traditional healers and the herbalists in all the selected communities for the purpose of obtaining their consent. The need to provide accurate, up to date, detailed and first-hand information as a way to achieve proper documentation of the indigenous medicinal plants of value to their health and sustenance was also emphasised. The discussions and interviews conducted were structured according to the standardized enquiry list of the PROTEA (Plant resources of Tropical Africa) DATABANK online database sheet (PROTABASE LINK). PROTA is an initiative of Wageningen University, the Netherlands working in cooperation with a variety of institutes in Africa and Europe. PROTA synthesizes all the information available for approximately 8,000 plants used in tropical Africa and makes it widely accessible in various media. Researchers asked the questions about prescriptions techniques; i.e, which species of plant is used for the treatment of particular ailments. Answers to these questions afford researchers the opportunity to generate detailed information on the ailments and plant parts used. Other questions that were asked include sources/places of collection of the plants or parts used, methods of application/administration, unit of measurement of the dosage and price, prevailing ailment in these communities, and accessibility to health care facilities. Method of knowledge transfer from the herbal vendors and herbalists to the young ones was established by engaging their biological children as apprentice. All the interviews and discussions were conducted in local Yoruba language. There was no problem of communication since the researchers are from the Yoruba speaking part of the country. No plant sample was collected as all the identifications were carried out on site based on the experience of the researchers (Oladeji and Adeyemo 2012). This was complemented with knowledgeable source (the researchers being accompanied by the assigned botanist from the research unit of Old Oyo National Park who incidentally is an indigene that has been practicing traditional healing over the years). Relevant literatures were also consulted as field guide books (Arbonnier 2004; Keay1959; Keay et al., 1964 and Odugbemi 2008). Some of these books contain coloured pictures of flora species (trees, shrubs and herbaceous plants) notable to these areas. These books were taken along and shown to the respondents in order to facilitate identification, especially when the key parts such as flowers, fruits and seeds that facilitate identification have been detached.

\section{Focus Group Discussion (FGD)}

FGD was held with a group of herbal vendors selected in each of the major commercial markets in the four LGAs (Iseyin, Atiba, Saki East and Atisbo) making a total of four group discussions held in the course of the study. The relationship between herbal vendors and herbalist is like that of pharmacists and the doctor in contemporary medicine. While the doctors diagnose and prescribe, the pharmacists are regarded as the chemists who interpret the composition of the prescription and the physical properties. After the patient has been diagnosed for the ailments by the herbalist, herbal vendors would be consulted to provide information on the chemistry (composition and physical properties) of the medicinal plants that are prescribed by the herbalists. This information led to the application or administration of the medicinal plants in form of concoction, decoction and body lotion or powdered as the case may be. The number of participants selected for the Focus Group Discussion varies from 10 to 13, depending on the total number of herbal vendors encountered in each commercial market and the number of years of selling herbal medicine, i.e 13 in Iseyin,, 12 in Atiba ; 10 in Saki East and in Atisbo. The participants were in the age range of 39-45 with females constituting the greater percentage. Apprentices were also included in the survey. The apprentices were said to be learning the usage and marketing of herbal plants or parts of it for an agreed period of time.

\section{Key Informants Interviewed}

Traditional healers and herbalists served as key informants, and Snowball sampling technique was used for selecting key informants for the interview. This technique involved locating the heads of the herbalists in the communities in order to collect the list of registered members and their contact addresses. Contact addresses of 16 members who were known to have spent up to twenty years or more in the practice of traditional medicine were taken from a list of fifty two registered members as the key informants. Considering length of time they spend in the practice of traditional medicine. Key informants are expected to have a profound knowledge of useful medicinal plants including familiarity with the culture, tradition, belief and norms of these communities. The informants were asked to mention different ailments in the selected communities along with plants or parts being used for the treatment of these ailments. Other information gathered from the key informants included source of collection and process of traditional knowledge transfer from one generation to the other. Male and female apprentices were encountered at the herbalists' home which is an indication of how traditional knowledge is passed from one generation to the other. Meanwhile, male practitioners constituted greater percentage $(87.5 \%)$ of the key informants. This also shows that two of the sixteen selected informants are female.

\section{Field observation}

Field observation was carried out in home gardens established at the back yards of the traditionalists/ herbalists/diviners where some identified medicinal plant species were grown to ensure that their immediate needs were met. Cultivated farmlands and the forest area adjoining the communities were also visited. Diversity of species of medicinal 
plants in the forest area is documented by Oladeji and Adeyemo (2012). Lists of species of medicinal plants observed in the cultivated farm lands and home gardens were also documented in the present study.

\section{Data Analysis}

Uses of plants as discussed during small group and individual interviews were arranged according to plant usage categories developed by Moerman (1998) and modified by Kassam et al. (2010). Kassam et al., (2010) developed a grouping based on the use of medicinal plants among indigenous Pamir Mountains of Afghanistan and Tajikistan. In cases where a particular use of a plant could be assigned to more than one category, the most specific category was used; i.e, no description of use was assigned to more than one category. The researchers carried out modifications and additions to the categories of Kassam et al. (2010) in order to reflect the context specificity of medicinal uses described by the host communities of Old Oyo National Park. Three categories were modified and ten new medicinal plant uses were added for treatment and prevention based on the context of the host communities of Old Oyo National Park. Medicinal plants per function were categorised rather than medicinal plants per species based on the application of plant usage categories and in some instances at the discretion of the researchers. This method offers two opportunities to examine medicinal plants per function rather than per species, to allow for comparison with current trends in the institutional health care system to evaluate the potential for medicinal plants to improve human health. The data obtained were analysed relative to the demographic characteristics of the respondents. 
Table1. Names of plants used for economic and medicinal purposes, parts being used, method of use and ailments being treated with

\begin{tabular}{|c|c|c|c|c|c|c|c|}
\hline $\mathrm{S} / \mathrm{N}$ & Scientific Name & Family & Local name & $\begin{array}{l}\text { Parts being } \\
\text { utilized }\end{array}$ & $\begin{array}{l}\text { Method } \\
\text { application }\end{array}$ & $\begin{array}{l}\text { Ailments } \\
\text { being } \\
\text { treated }\end{array}$ & $\begin{array}{l}\text { Conservation status } \\
\text { IUCN/Catalogue of life }\end{array}$ \\
\hline 1 & $\begin{array}{l}\text { Abrus precatorius L. (climbing } \\
\text { herb) }\end{array}$ & $\begin{array}{l}\text { LEGUMINOSA } \\
\text { E }\end{array}$ & Ojuologbo & Leave & Decoction & Cough & ++ \\
\hline 2 & $\begin{array}{l}\text { Acacia ataxacantha DC. } \\
\text { (scrambling shrub) }\end{array}$ & $\begin{array}{l}\text { MIMOSOIDEA } \\
\text { E }\end{array}$ & Cassia & Root & Decoction & Dysentery & ++ \\
\hline 3 & $\begin{array}{l}\text { Acacia nilotica(L.) Wild. ex Del } \\
\text { (Tree) }\end{array}$ & $\begin{array}{l}\text { MIMOISOIDE } \\
\mathrm{AE}\end{array}$ & Booni & Bark & Fumigation & $\begin{array}{l}\text { Fungal } \\
\text { fumigant }\end{array}$ & ++ \\
\hline 4 & Adasonia digitataL. (Tree) & $\begin{array}{l}\text { BOMBACACE } \\
\text { AE }\end{array}$ & Ose & Bark & $\begin{array}{l}\text { Concoction cook } \\
\text { with aethiopia }\end{array}$ & $\begin{array}{l}\text { For baby } \\
\text { to quickly } \\
\text { gain } \\
\text { weight or } \\
\text { to treat } \\
\text { caries in } \\
\text { children }\end{array}$ & ++ \\
\hline 5 & $\begin{array}{l}\text { Aframonumm elegueta Rosc } \\
\text { (Herb) }\end{array}$ & $\begin{array}{l}\text { ZINGIBERACE } \\
\mathrm{AE}\end{array}$ & Atare & Leave & Decoction & $\begin{array}{l}\text { Diarrhea, } \\
\text { skin } \\
\text { diseases. } \\
\text { Malaria, } \\
\text { dietary aid }\end{array}$ & ++ \\
\hline 6 & Ageratum conyzoides L. (Herb) & ASTERACEAE & Imi-esu & $\begin{array}{l}\text { Fruit and } \\
\text { seed }\end{array}$ & Decoction & $\begin{array}{l}\text { dysentery } \\
\text { malaria, } \\
\text { stimulant }\end{array}$ & $* *$ \\
\hline 7 & Allium ascalonicum L. (Herb) & LILIACEAE & Alubosa-elewe & Leaves & Decoction & $\begin{array}{l}\text { Malaria, } \\
\text { cough, }\end{array}$ & $* *$ \\
\hline 8 & Allium sativum L.(Herb) & LILIACEAE & Alubosa-ayu & Bulb & $\begin{array}{l}\text { Decoction, } \\
\text { ingredient }\end{array}$ & $\begin{array}{l}\text { Malaria, } \\
\text { cough, } \\
\text { preservativ } \\
\text { e }\end{array}$ & $* *$ \\
\hline 9 & Alternathera repens L. (Herb) & $\begin{array}{l}\text { AMARANTHA } \\
\text { CEAE }\end{array}$ & Dagunro & Leave & $\begin{array}{l}\text { Decoction, } \\
\text { Dysentery }\end{array}$ & $\begin{array}{l}\text { Dewormin } \\
\text { g, Malaria } \\
\text { fever. }\end{array}$ & ++ \\
\hline 10 & $\begin{array}{l}\text { Anarcadium occidentale L. } \\
\text { (Tree) }\end{array}$ & $\begin{array}{l}\text { ANACARDIAC } \\
\text { EAE }\end{array}$ & Kaju & $\begin{array}{l}\text { Leave, } \\
\text { Bark }\end{array}$ & Concoction & Malaria & ++ \\
\hline 11 & $\begin{array}{l}\text { Annona } \quad \text { senegalensisPers. } \\
\text { (Tree) }\end{array}$ & $\begin{array}{l}\text { ANNONACEA } \\
\text { E }\end{array}$ & Abo & Bark, leave & Concoction & $\begin{array}{l}\text { Diabetics, } \\
\text { venereal } \\
\text { disease, } \\
\text { gonorrhea, } \\
\text { watery } \\
\text { sperm }\end{array}$ & $* *$ \\
\hline 12 & $\begin{array}{l}\text { Anogeissus } \\
\text { leiocarpus (DC.) Guill. \&Perr. }\end{array}$ & $\begin{array}{l}\text { COMBRETAC } \\
\text { EAE }\end{array}$ & Ayin & Root, bark & $\begin{array}{l}\text { Chewing stick, } \\
\text { concoction }\end{array}$ & $\begin{array}{l}\text { Mouth } \\
\text { disease, }\end{array}$ & ++ \\
\hline
\end{tabular}




\begin{tabular}{|c|c|c|c|c|c|c|c|}
\hline $\mathrm{S} / \mathrm{N}$ & Scientific Name & Family & Local name & $\begin{array}{l}\text { Parts being } \\
\text { utilized }\end{array}$ & $\begin{array}{l}\text { Method } \\
\text { application }\end{array}$ & $\begin{array}{l}\text { Ailments } \\
\text { being } \\
\text { treated }\end{array}$ & $\begin{array}{l}\text { Conservation status } \\
\text { IUCN/Catalogue of life }\end{array}$ \\
\hline 13 & $\begin{array}{l}\text { (Tree) } \\
\text { Anthocleista djalonesis A. } \\
\text { Chev. (Tree) }\end{array}$ & $\begin{array}{l}\text { LOGANIACEA } \\
\text { E }\end{array}$ & Sapo & Bark & Concoction & $\begin{array}{l}\text { dysentery } \\
\text { Skin } \\
\text { disease, } \\
\text { malaria }\end{array}$ & ++ \\
\hline 14 & $\begin{array}{ll}\text { Aristolochia } & \text { ringens Vahl } \\
\text { (Twining herb) } & \end{array}$ & $\begin{array}{l}\text { ARISTOLOCHI } \\
\text { ACEAE }\end{array}$ & Akogun & Root & Decoction & $\begin{array}{l}\text { Typhoid, } \\
\text { malaria }\end{array}$ & $* *$ \\
\hline 15 & $\begin{array}{l}\text { Azadirachta indicaA. Juss } \\
\text { (Tree) }\end{array}$ & MELIACEAE & Dangoyaro & $\begin{array}{l}\text { Leave, } \\
\text { bark }\end{array}$ & Concoction & Malaria & ++ \\
\hline 16 & Basella alba L.(Creeping herb) & $\begin{array}{l}\text { BASELLACEA } \\
\text { E }\end{array}$ & Amunututu & Leave & Decoction & $\begin{array}{l}\text { Stomach } \\
\text { disturbanc } \\
\text { e }\end{array}$ & $* *$ \\
\hline 17 & $\begin{array}{l}\text { Berlinia grandiflora (Vahl) } \\
\text { Hutch. \&Dalz. (Tree) }\end{array}$ & $\begin{array}{l}\text { CAESALPINIO } \\
\text { IDEAE }\end{array}$ & Apado & Latex & & $\begin{array}{l}\text { To } \\
\text { increase } \\
\text { man's } \\
\text { libido }\end{array}$ & ++ \\
\hline 18 & Blighia sapida Koenig (Tree) & $\begin{array}{l}\text { SAPINDACEA } \\
\text { E }\end{array}$ & Isin & Fruit & Soup condiment & $\begin{array}{l}\text { Dysentery, } \\
\text { diabetes }\end{array}$ & $* *$ \\
\hline 19 & $\begin{array}{l}\text { Bridelia ferruginea Benth. } \\
\text { (Tree) }\end{array}$ & $\begin{array}{l}\text { EUPHORBIAC } \\
\text { EAE }\end{array}$ & Ira & $\begin{array}{l}\text { Leave, } \\
\text { stem }\end{array}$ & $\begin{array}{l}\text { Decoction } \\
\text { chewing stick }\end{array}$ & $\begin{array}{l}\text { Dysentery, } \\
\text { teeth } \\
\text { disease, } \\
\text { antibacteri } \\
\text { al, malaria. }\end{array}$ & $* *$ \\
\hline 20 & $\begin{array}{l}\text { Bridelia micrantha (Hochst.) } \\
\text { Baill (Tree) }\end{array}$ & $\begin{array}{l}\text { EUPHORBIAC } \\
\text { EAE }\end{array}$ & Isa & $\begin{array}{l}\text { Leave, } \\
\text { fruit }\end{array}$ & Concoction & $\begin{array}{l}\text { Yellow } \\
\text { fever, } \\
\text { jaundice }\end{array}$ & $* *$ \\
\hline 21 & Cajanus cajan Harms (Shrub) & $\begin{array}{l}\text { CAESALPINIO } \\
\text { IDEAE }\end{array}$ & Otili & $\begin{array}{l}\text { Leave, } \\
\text { seed }\end{array}$ & $\begin{array}{l}\text { Concoction, Cook } \\
\text { the seed }\end{array}$ & $\begin{array}{l}\text { Malaria, } \\
\text { protein } \\
\text { constituent }\end{array}$ & ++ \\
\hline 22 & Capsicum frutescens L. (Shrub) & SOLANACEAE & Ata were & Seed, fruit & $\begin{array}{l}\text { Soup condiment, } \\
\text { concoction }\end{array}$ & $\begin{array}{l}\text { Stimulant, } \\
\text { laryngitis }\end{array}$ & ++ \\
\hline 23 & Carica papaya L. (Tree) & $\begin{array}{l}\text { CAPPARACEA } \\
\text { E }\end{array}$ & Ibepe & $\begin{array}{l}\text { Leave, } \\
\text { fruit }\end{array}$ & $\begin{array}{l}\text { Decoction and } \\
\text { concoction }\end{array}$ & $\begin{array}{l}\text { Malaria, } \\
\text { jaundice }\end{array}$ & $* *$ \\
\hline 24 & Cassia sieberiana DC. (Tree) & CARICACEAE & Aidan tooro & Root & Concoction & Dysentery & ++ \\
\hline 25 & Celosia laxa Auct. (Herb) & $\begin{array}{l}\text { AMARANTHA } \\
\text { CEAE }\end{array}$ & $\begin{array}{l}\text { Marugbo- } \\
\text { sanyan }\end{array}$ & Leave & $\begin{array}{l}\text { Concoction, soup } \\
\text { condiment }\end{array}$ & $\begin{array}{l}\text { Breast pain } \\
\text { in women } \\
\text { (abscesses } \\
\text { of breast) }\end{array}$ & ++ \\
\hline 26 & $\begin{array}{l}\text { Chasmanthera dependens } \\
\text { Hochst. (Creeping Herb) }\end{array}$ & $\begin{array}{l}\text { MENISPERMA } \\
\text { CEAE }\end{array}$ & Atoo & $\begin{array}{l}\text { Leave, } \\
\text { bark }\end{array}$ & $\begin{array}{l}\text { Decoction, grind } \\
\text { the leave and mix } \\
\text { with body } \\
\text { ointment }\end{array}$ & $\begin{array}{l}\text { Fracture, } \\
\text { swollen leg } \\
\text { or wound, } \\
\text { children }\end{array}$ & ++ \\
\hline
\end{tabular}




\begin{tabular}{|c|c|c|c|c|c|c|c|}
\hline $\mathrm{S} / \mathrm{N}$ & Scientific Name & Family & Local name & $\begin{array}{l}\text { Parts being } \\
\text { utilized }\end{array}$ & $\begin{array}{l}\text { Method } \\
\text { application }\end{array}$ & $\begin{array}{l}\text { Ailments } \\
\text { being } \\
\text { treated }\end{array}$ & $\begin{array}{l}\text { Conservation status } \\
\text { IUCN/Catalogue of life }\end{array}$ \\
\hline 27 & $\begin{array}{l}\text { Chenopodium ambrosioides } \\
\text { L.(Herb) }\end{array}$ & $\begin{array}{l}\text { CHENOPODIA } \\
\text { CEAE }\end{array}$ & Orugbon & Leave & $\begin{array}{l}\text { Mix the } \\
\text { with } \\
\text { ointment }\end{array}$ & $\begin{array}{l}\text { with weak } \\
\text { bones } \\
\text { Arthritis, } \\
\text { wound }\end{array}$ & ++ \\
\hline 28 & $\begin{array}{l}\text { Citrus aurantifolia (Christm.) } \\
\text { Swingle (Tree) }\end{array}$ & RUTACEAE & Osanoronbo & $\begin{array}{l}\text { Fruit, } \\
\text { leave, bark }\end{array}$ & $\begin{array}{l}\text { Decoction } \\
\text { concoction }\end{array}$ & $\begin{array}{l}\text { Typhoid, } \\
\text { malaria, } \\
\text { dysentery }\end{array}$ & ++ \\
\hline 29 & Cocos nucifera L. (Tree) & PALMAE & Agbon & Nuts, bark & $\begin{array}{l}\text { Decoction, fruit is } \\
\text { edible }\end{array}$ & $\begin{array}{l}\text { Skin } \\
\text { disease, } \\
\text { uterine } \\
\text { disease }\end{array}$ & $* *$ \\
\hline 30 & $\begin{array}{l}\text { Combretum molle R.Br. ex G. } \\
\text { Don (Tree) }\end{array}$ & $\begin{array}{l}\text { COMBRETAC } \\
\text { EAE }\end{array}$ & Okuku & Bark & Concoction & Dysentery & ++ \\
\hline 31 & Crinum jagus Thombs.(Herb) & $\begin{array}{l}\text { AMARYLLID } \\
\text { ACEAE }\end{array}$ & Ireke-odo & Root & Concoction & $\begin{array}{l}\text { Convulsio } \\
\mathrm{n} \text {, cough }\end{array}$ & $* *$ \\
\hline 32 & $\begin{array}{l}\text { Cucumis melo L.(Creeping } \\
\text { Herb) }\end{array}$ & $\begin{array}{l}\text { CUCURBITAC } \\
\text { EAE }\end{array}$ & Baara & Fruit & $\begin{array}{l}\text { Decoction in a } \\
\text { fermented maize } \\
\text { corn water }\end{array}$ & $\begin{array}{l}\text { Gonorrhea, } \\
\text { dysentery }\end{array}$ & $* *$ \\
\hline 33 & Cyathula prostrata B1. (Herb) & $\begin{array}{l}\text { AMARANTHA } \\
\text { CEAE }\end{array}$ & Soworepepe & Leave & Concoction & $\begin{array}{l}\text { To boost } \\
\text { trading or } \\
\text { business }\end{array}$ & ++ \\
\hline 34 & 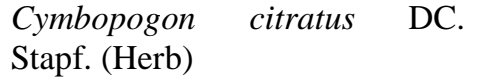 & POACEAE & Ewe tea & Leave & Concoction & $\begin{array}{l}\text { Malaria } \\
\text { fever }\end{array}$ & ++ \\
\hline 35 & $\begin{array}{l}\text { Diocleare } \quad \text { flexa } \quad \text { Hook. } \\
\text { (Climber) }\end{array}$ & $\begin{array}{l}\text { LEGUNINOSA } \\
\mathrm{E}\end{array}$ & Agbaarin & Seed & Decoction & $\begin{array}{l}\text { To } \\
\text { improve } \\
\text { Woman } \\
\text { fertility }\end{array}$ & ++ \\
\hline 36 & Enantiac hlorantha Oliv. (Tree) & $\begin{array}{l}\text { ANNONACEA } \\
\text { E }\end{array}$ & $\begin{array}{l}\text { Awopa or } \\
\text { bush doctor }\end{array}$ & Bark & Decoction & Malaria & ++ \\
\hline 37 & $\begin{array}{l}\text { Entada Africana Guilli. \&Perr. } \\
\text { (Tree) }\end{array}$ & $\begin{array}{l}\text { LEGUMINOSA } \\
\text { E }\end{array}$ & Igbanso & $\begin{array}{l}\text { Bark, root, } \\
\text { leave }\end{array}$ & Concoction & Diarrhea & $* *$ \\
\hline 38 & $\begin{array}{ll}\text { Erythrococca } & \text { anomala } \\
\text { Juss.ex.Purr (Tree) } & \end{array}$ & $\begin{array}{l}\text { EUPHORBIAC } \\
\text { EAE }\end{array}$ & Iyere & Fruit, seed & $\begin{array}{l}\text { Decoction, spices } \\
\text { soup }\end{array}$ & $\begin{array}{l}\text { Dysentery, } \\
\text { stimulant }\end{array}$ & ++ \\
\hline 39 & $\begin{array}{l}\text { Eugenia aromatic } \quad \text { Kuntze } \\
\text { (shrub) }\end{array}$ & MYRTACEAE & Kanafuru & Seed & $\begin{array}{l}\text { Decoction, soup } \\
\text { spices, } \\
\text { preservative }\end{array}$ & $\begin{array}{l}\text { Dysentery, } \\
\text { diarrhea }\end{array}$ & $* *$ \\
\hline 40 & $\begin{array}{ll}\text { Euphorbia laterifolia } & \text { lam } \\
\text { Schum\&Thun (Herb) }\end{array}$ & $\begin{array}{l}\text { EUPHORBIAC } \\
\text { EAE }\end{array}$ & Enuopiye & $\begin{array}{l}\text { Stem, } \\
\text { leaves }\end{array}$ & $\begin{array}{l}\text { Decoction, grind } \\
\text { and mix with } \\
\text { body ointment }\end{array}$ & $\begin{array}{l}\text { Skin } \\
\text { disease, } \\
\text { hives }\end{array}$ & ++ \\
\hline 41 & Erythrophleum & & Erun obo & Bark & Decoction, burn & Repellent & \\
\hline
\end{tabular}




\begin{tabular}{|c|c|c|c|c|c|c|c|}
\hline $\mathrm{S} / \mathrm{N}$ & Scientific Name & Family & Local name & $\begin{array}{l}\text { Parts being } \\
\text { utilized }\end{array}$ & $\begin{array}{l}\text { Method } \\
\text { application }\end{array}$ & $\begin{array}{l}\text { Ailments } \\
\text { being } \\
\text { treated }\end{array}$ & $\begin{array}{l}\text { Conservation status } \\
\text { IUCN/Catalogue of life }\end{array}$ \\
\hline & (Guill\&Perr.) Brenan (Tree) & $\begin{array}{l}\text { CAESALPINIO } \\
\text { IDEAE }\end{array}$ & & & as incense & $\begin{array}{ll}\text { of } & \text { evil } \\
\text { spirit } & \end{array}$ & ++ \\
\hline 42 & Ficus capensis Thunb (Tree) & MORACEAE & Opoto & $\begin{array}{l}\text { Stem, leaf, } \\
\text { root }\end{array}$ & Concoction & $\begin{array}{l}\text { Gonorrhea, } \\
\text { dysentery }\end{array}$ & ++ \\
\hline 43 & $\begin{array}{l}\text { Funtumia elastic ( Preuss) } \\
\text { Stapf. (Tree) }\end{array}$ & $\begin{array}{l}\text { APOCYNACE } \\
\text { AE }\end{array}$ & Ire & Bark & $\begin{array}{l}\text { Soak in fermented } \\
\text { water from } \\
\text { grounded maize }\end{array}$ & $\begin{array}{l}\text { Diarrhea, } \\
\text { dysentery }\end{array}$ & $* *$ \\
\hline 44 & Garcinia kola Heckel (Tree) & GUTTIFERAE & Orogbo & $\begin{array}{l}\text { Seeds, } \\
\text { root, bark }\end{array}$ & $\begin{array}{l}\text { Chewing the seed, } \\
\text { decoction }\end{array}$ & $\begin{array}{l}\text { Cough, } \\
\text { bronchitis, } \\
\text { throat and } \\
\text { respiratory } \\
\text { disorder }\end{array}$ & $\mathrm{V}$ \\
\hline 45 & $\begin{array}{l}\text { Hannoa undulate (Guill. } \\
\text { \&Perr.) Planch.(Herb) }\end{array}$ & GUTTIFERAE & Ewe oriji & Leave, root & $\begin{array}{l}\text { Decoction } \\
\text { concoction }\end{array}$ & $\begin{array}{l}\text { To receive } \\
\text { favour or } \\
\text { forgivenes } \\
\mathrm{s} \text {, restore } \\
\text { broken } \\
\text { love or } \\
\text { relationshi } \\
\mathrm{p} \text { to boost } \\
\text { sales }\end{array}$ & ++ \\
\hline 46 & $\begin{array}{l}\text { Harunganamadaga } \\
\text { Lam. ex Poir (Tree) }\end{array}$ & GUTTIFERA & Amuje & Bark, leave & Concoction & $\begin{array}{l}\text { antianaemi } \\
\text { a }\end{array}$ & ++ \\
\hline 47 & $\begin{array}{l}\text { Kalanchoe crenata (Andrews } \\
\text { Haw) (Shrub) }\end{array}$ & $\begin{array}{l}\text { CRASSULACE } \\
\text { AE }\end{array}$ & Odundun & Leave & Concoction & $\begin{array}{l}\text { Gonorrhea, } \\
\text { small pox, } \\
\text { to boost } \\
\text { trading }\end{array}$ & ** \\
\hline 48 & $\begin{array}{l}\text { Khaya senegalensis (Desr.) } \\
\text { A.Juss. (Tree) }\end{array}$ & MELIACEAE & Oganwo & Bark & $\begin{array}{l}\text { Decoction, } \\
\text { concoction }\end{array}$ & $\begin{array}{l}\text { Skin } \\
\text { disease, } \\
\text { malaria }\end{array}$ & $\mathrm{V}$ \\
\hline 49 & $\begin{array}{l}\text { Kigelia africana( Lam.) Benth. } \\
\text { (Tree) }\end{array}$ & $\begin{array}{l}\text { BIGNONIACE } \\
\mathrm{AE}\end{array}$ & Pandoro & $\begin{array}{l}\text { Bark, stem, } \\
\text { root, fruit }\end{array}$ & Concoction & $\begin{array}{l}\text { Malaria, } \\
\text { dysentery, } \\
\text { gonorrhea, } \\
\text { rheumatis } \\
\text { m }\end{array}$ & $* *$ \\
\hline 50 & $\begin{array}{l}\text { Lagenaria breviflorus (Benth) } \\
\text { Roberty (Creeping Herb) }\end{array}$ & $\begin{array}{l}\text { CUCURBITAC } \\
\text { EAE }\end{array}$ & Tangiri & Fruit & $\begin{array}{l}\text { Decoction, throw } \\
\text { on the floor } \\
\text { around the house } \\
\text { to prevent spread } \\
\text { of small pox }\end{array}$ & $\begin{array}{l}\text { Small pox, } \\
\text { purgative }\end{array}$ & ++ \\
\hline 51 & Lagenaria siceraria (Molina) & CUCURBITAC & Ado & Fruit pup & Dried fruit pup as & Anthelmint & $* *$ \\
\hline
\end{tabular}




\begin{tabular}{|c|c|c|c|c|c|c|c|}
\hline $\mathrm{S} / \mathrm{N}$ & Scientific Name & Family & Local name & $\begin{array}{l}\text { Parts being } \\
\text { utilized }\end{array}$ & $\begin{array}{l}\text { Method of } \\
\text { application }\end{array}$ & $\begin{array}{l}\text { Ailments } \\
\text { being } \\
\text { treated }\end{array}$ & $\begin{array}{l}\text { Conservation status } \\
\text { IUCN/Catalogue of life }\end{array}$ \\
\hline & Standl. cv (Creeping Herb) & EAE & & & $\begin{array}{l}\text { gourd to keep } \\
\text { herbal medicine }\end{array}$ & $\begin{array}{l}\text { ic } \\
\text { properties }\end{array}$ & \\
\hline 52 & $\begin{array}{l}\text { Launaea taraxacifolia (Willd) } \\
\text { Amin Ex. C. Jeffry (Herb) }\end{array}$ & ASTERACEAE & Yanrin & Leave & Decoction & Cataract & ++ \\
\hline 53 & $\begin{array}{l}\text { Lippia multiflora Moldenke } \\
\text { (Herb) }\end{array}$ & $\begin{array}{l}\text { VERBENACE } \\
\text { AE }\end{array}$ & $\begin{array}{l}\text { Efinrin- } \\
\text { foronoba }\end{array}$ & Leave & Decoction & Dysentery & ++ \\
\hline 54 & Mangifera indicaL. (Tree) & $\begin{array}{l}\text { ANACARDIAC } \\
\text { EAE }\end{array}$ & Mangoro & $\begin{array}{l}\text { Leave, } \\
\text { bark, fruit }\end{array}$ & Concoction & $\begin{array}{l}\text { Malaria, } \\
\text { juice } \\
\text { production }\end{array}$ & $\mathrm{D}$ \\
\hline 55 & $\begin{array}{l}\text { Melicia excelsa (Welw.) CC } \\
\text { Berg. (Tree) }\end{array}$ & MELIACEAE & Iroko & $\begin{array}{l}\text { Root, fruit, } \\
\text { seed, bark }\end{array}$ & Concoction & $\begin{array}{l}\text { Neutralizer } \\
\text {, spiritual } \\
\text { deliveranc } \\
\text { e from } \\
\text { oppression }\end{array}$ & ++ \\
\hline 56 & Morinda lucida Benth. (Tree) & RUBIACEAE & Oruwo & Leave & Concoction & Malaria & $* *$ \\
\hline 57 & $\begin{array}{l}\text { Nauclea latifolia ( Smith) } \\
\text { Bruce (Tree) }\end{array}$ & RUBIACEAE & Egbesi & Leave, root & $\begin{array}{l}\text { Concoction or } \\
\text { decoction (soak } \\
\text { the root in water } \\
\text { with Eugenia } \\
\text { aromatica) }\end{array}$ & Malaria & $* *$ \\
\hline 58 & Ocinum basilicum L. (Herb) & LABIATAE & Efinrin & Leave & Decoction & $\begin{array}{l}\text { Dysentery, } \\
\text { loss of } \\
\text { appetite, } \\
\text { sleeplessne } \\
\text { ss }\end{array}$ & ++ \\
\hline 59 & Olax subscorpiodea Oliv. (Tree) & OLACACEAE & Ifun & Root & Concoction & $\begin{array}{l}\text { Malaria, } \\
\text { yellow } \\
\text { fever }\end{array}$ & ++ \\
\hline 60 & $\begin{array}{l}\text { Parinari curatellifolia Planch. } \\
\text { exBenth. (Tree) }\end{array}$ & ROSACEAE & Idofin & Root, bark & $\begin{array}{l}\text { Powdered the } \\
\text { bark (Agunmu) } \\
\text { root can be } \\
\text { chewed }\end{array}$ & $\begin{array}{l}\text { Skin } \\
\text { disease, } \\
\text { cough, } \\
\text { dysentery }\end{array}$ & ++ \\
\hline 61 & $\begin{array}{l}\text { Parkia biglobosa (Jacq.) R. Br. } \\
\text { ex G. Don(Tree) }\end{array}$ & $\begin{array}{l}\text { MIMOSOIDEA } \\
\mathrm{E}\end{array}$ & Igiigba & $\begin{array}{l}\text { Bark, } \\
\text { leave, } \\
\text { seeds, fruit } \\
\text { pup }\end{array}$ & $\begin{array}{l}\text { Soup condiment } \\
\text { (Iru), concoction }\end{array}$ & $\begin{array}{l}\text { Tonic, } \\
\text { malaria, } \\
\text { intestinal } \\
\text { and mental } \\
\text { disorder }\end{array}$ & $* *$ \\
\hline 62 & $\begin{array}{l}\text { Peperomiape llucida L.HBK } \\
\text { (Herb) }\end{array}$ & PIPERACEAE & Rinrin & Leave & $\begin{array}{l}\text { Mix the leave } \\
\text { with ointment } \\
\text { (Ori) }\end{array}$ & Small pox & $* *$ \\
\hline 63 & Phyllanthus & EUPHORBIAC & Eyinolobe & Leave & Grind the leave & Hives, & $* *$ \\
\hline
\end{tabular}




\begin{tabular}{|c|c|c|c|c|c|c|c|}
\hline $\mathrm{S} / \mathrm{N}$ & Scientific Name & Family & Local name & $\begin{array}{l}\text { Parts being } \\
\text { utilized }\end{array}$ & $\begin{array}{l}\text { Method } \\
\text { application }\end{array}$ & $\begin{array}{l}\text { Ailments } \\
\text { being } \\
\text { treated }\end{array}$ & $\begin{array}{l}\text { Conservation status } \\
\text { IUCN/Catalogue of life }\end{array}$ \\
\hline & Schum\&Thonn (Herb) & EAE & & & $\begin{array}{l}\text { and mix with } \\
\text { ointment (Ori) }\end{array}$ & small pox & \\
\hline 64 & $\begin{array}{l}\text { Piliostigma thonningii } \\
\text { (Schumach.) Milne-Redh. } \\
\text { (Tree) }\end{array}$ & $\begin{array}{l}\text { CAESALPINIA } \\
\text { CEAE }\end{array}$ & Abafe & $\begin{array}{l}\text { Root, bark, } \\
\text { leave }\end{array}$ & Concoction & $\begin{array}{l}\text { Malaria, } \\
\text { diabetes }\end{array}$ & ++ \\
\hline 65 & $\begin{array}{l}\text { Pseudocedrela kotschyi } \\
\text { (Schweinf.) Harms (Tree) }\end{array}$ & MELIACEAE & Emi gbegi & Bark, root & $\begin{array}{l}\text { Chewing stick, } \\
\text { concoction }\end{array}$ & $\begin{array}{l}\text { Skin } \\
\text { disease, } \\
\text { dysentery }\end{array}$ & ++ \\
\hline 66 & $\begin{array}{l}\text { Securidaca } \\
\text { longepedunculataFres. (Tree) }\end{array}$ & $\begin{array}{l}\text { POLYGALACE } \\
\text { AE }\end{array}$ & Ipeta & Root & Decoction & $\begin{array}{l}\text { Venereal } \\
\text { disease, } \\
\text { children } \\
\text { skin } \\
\text { disease }\end{array}$ & ++ \\
\hline 67 & $\begin{array}{l}\text { Securine gavirosa } \\
\text { (Roxb,exWilld.) Baill.(Tree) }\end{array}$ & $\begin{array}{l}\text { EUPHORBIAC } \\
\text { EAE }\end{array}$ & Iranje & Root & Decoction & $\begin{array}{l}\text { Diarrhea, } \\
\text { dysentery, } \\
\text { jaundice }\end{array}$ & $* *$ \\
\hline 68 & $\begin{array}{l}\text { Schebera arborea A. Chev. } \\
\text { (Tree) }\end{array}$ & OLEACEAE & Opele & Bark & Concoction & Fontanel & ++ \\
\hline 69 & $\begin{array}{l}\text { Senna hirsuta (L.) Irwin and } \\
\text { Barneby (Herb) }\end{array}$ & $\begin{array}{l}\text { CAESALPINIO } \\
\text { IDEAE }\end{array}$ & Amunimuye & Leave, root & Decoction & $\begin{array}{l}\text { Skin } \\
\text { diseases, } \\
\text { syphilis }\end{array}$ & ++ \\
\hline 70 & $\begin{array}{l}\text { Senecio abyssinicus Sch. Bip. } \\
\text { ex-A Rich (Shrub) }\end{array}$ & $\begin{array}{l}\text { LEGUMINOSA } \\
\text { E }\end{array}$ & Rere & Leave & Decoction & Purgative & ++ \\
\hline 71 & $\begin{array}{l}\text { Sorghum bicolor (L.) Moench } \\
\text { (herb) }\end{array}$ & POACEAE & Epolola & Leave & Concoction & $\begin{array}{l}\text { Anemia } \\
\text { (Serve as } \\
\text { blood } \\
\text { tonic) }\end{array}$ & $* *$ \\
\hline 72 & $\begin{array}{l}\text { Sphenocentrum } \\
\text { Pierre (shrub) }\end{array}$ & $\begin{array}{l}\text { MENISPERMA } \\
\text { CEAE }\end{array}$ & Akerejupon & $\begin{array}{l}\text { Root } \\
\text { (yellow) }\end{array}$ & Concoction & $\begin{array}{l}\text { Malaria, } \\
\text { yellow } \\
\text { fever, } \\
\text { jaundice }\end{array}$ & ++ \\
\hline 73 & Spigelia anthelmia Linn (Tree) & $\begin{array}{l}\text { LOGANIACEA } \\
\text { E }\end{array}$ & Apaaran & Root & Concoction & $\begin{array}{l}\text { Anthelmint } \\
\text { ic , deworm }\end{array}$ & $* *$ \\
\hline 74 & $\begin{array}{l}\text { Terminalia macroptera } \\
\text { Guill.\&Perr. (Tree) }\end{array}$ & $\begin{array}{l}\text { COMBRETAC } \\
\text { EAE }\end{array}$ & Idi & $\begin{array}{l}\text { Root, } \\
\text { leave, bark }\end{array}$ & Chewing stick, & $\begin{array}{l}\text { Dysentery, } \\
\text { diarrhea }\end{array}$ & ++ \\
\hline 75 & $\begin{array}{l}\text { Tetrapleura tetraptera } \\
\text { (Schum.\&Thonn.) (Tree) }\end{array}$ & $\begin{array}{l}\text { MIMOSOIDEA } \\
\text { E }\end{array}$ & Aidan & Bark, fruit & $\begin{array}{lr}\text { Grind and } & \text { mix } \\
\text { local } & \text { soap } \\
\text { (Osedudu) } & \text { for } \\
\text { bathing, } & \\
\text { concoction } & \end{array}$ & $\begin{array}{l}\text { Skin } \\
\text { disease, } \\
\text { malaria }\end{array}$ & ++ \\
\hline 76 & Theobroma cacao L. (Tree) & STERCULIACE & Koko & Pod, fruit & Concoction, grind & Anemia & $* *$ \\
\hline
\end{tabular}




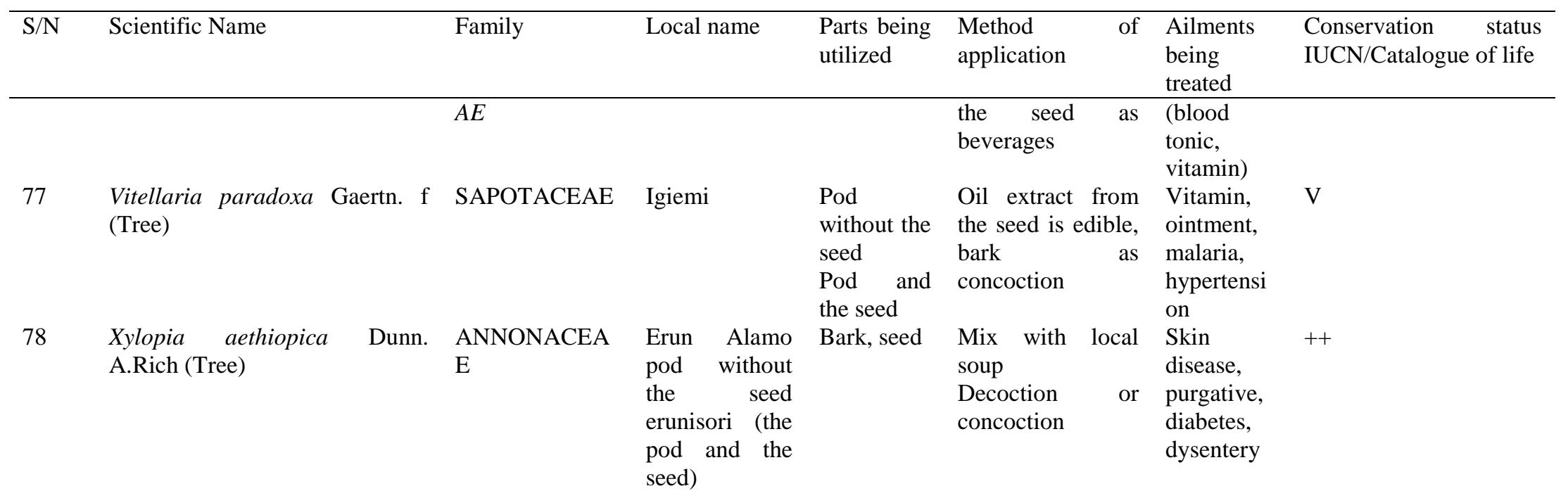


Note : ++- This taxon has not yet been assessed for the IUCN Red List, and also is not in the Catalogue of Life.

** - This taxon has not yet been assessed for the IUCN Red List, but is in the Catalogue of Life.

V- Vulnerable under IUCN category of conservation status

D-Data deficient and need updating

Table 2: Number and proportion of plants exclusively used for various categories of treatment and prevention.

\begin{tabular}{|c|c|c|}
\hline Category of medicinal use & Number of plants & Percent of all uses \\
\hline Cough medicine & 2 & 2.6 \\
\hline Dysentery medicine $^{\mathrm{a}}$ & 6 & 7.6 \\
\hline Incense/Evil spirit repellant ${ }^{\mathrm{b}}$ & 2 & 2.6 \\
\hline Pediatric $^{c}$ & 3 & 3.9 \\
\hline Dietary aid & 1 & 1.3 \\
\hline Stimulant & 2 & 2.6 \\
\hline Preservative & 1 & 1.3 \\
\hline Deworming/ Anthelmintic ${ }^{\mathrm{d}}$ & 3 & 3.9 \\
\hline Malaria & 7 & 8.8 \\
\hline Veneral disease $^{\mathrm{f}}$ & 6 & 7.6 \\
\hline Mouth/tooth disease ${ }^{\mathrm{g}}$ & 2 & 2.6 \\
\hline Dermatological aid ${ }^{\mathrm{h}}$ & 6 & 7.6 \\
\hline Typhoid & 2 & 2.6 \\
\hline Stomach disturbance ${ }^{\mathrm{i}}$ & 1 & 1.3 \\
\hline Man’s libido & 1 & 1.3 \\
\hline Diabetics medicine & 2 & 2.6 \\
\hline Yellow fever $^{j}$ & 4 & 5.0 \\
\hline Protein constituent & 1 & 1.3 \\
\hline Throat aid ${ }^{\mathrm{K}}$ & 1 & 1.3 \\
\hline Abscesses in breast of women & 1 & 1.3 \\
\hline Orthopedic $^{1}$ & 3 & 3.9 \\
\hline Gynecological aid & 2 & 2.6 \\
\hline Spiritual Intervention/deliverance ${ }^{\mathrm{m}}$ & 4 & 5.0 \\
\hline Antidiarrhe $^{\mathrm{n}}$ & 4 & 5.0 \\
\hline Respiratory disorder & 1 & 1.3 \\
\hline Antianaemia $^{\circ}$ & 3 & 3.9 \\
\hline Small pox & 2 & 2.6 \\
\hline Purgative & 2 & 2.6 \\
\hline Eye medicine & 1 & 1.3 \\
\hline Mental disorder & 1 & 1.3 \\
\hline Vitamin & 1 & 1.3 \\
\hline Total & 78 & 100 \\
\hline
\end{tabular}

${ }^{a}$ Medicinal Plants that treat dysentery, ${ }^{b}$ Medicinal plants that are burn to serve as incense to repel evil spirit from surrounding, ${ }^{\mathrm{c}}$ Modified from Kassam et al., 2010. Pediatric aid to include medicinal plants used in treating disease associated with children such as wide Fontanel, loss of weight or low weight gain, ${ }^{\mathrm{d}}$ Medicinal plants used in deworming ${ }^{\mathrm{e}}$ Medicinal plant used in treating malaria, ${ }^{\mathrm{f}}$ Medicinal plants used in treating venereal disease such as gonorrhea, syphilis ${ }^{\mathrm{g}}$ Medicinal plants used for the treatment of mouth and teeth disease, ${ }^{\mathrm{h}}$ Modified from Kassam et al., 2010 dermatological aid to include medicinal plant used in treating skin diseases in Adult and children, ${ }^{\mathrm{i}}$ Medicinal plant used in treating stomach disturbance, ${ }^{\mathrm{j}}$ Medicinal Plants used in the treatment of yellow fever and close to this jaundice, ${ }^{\mathrm{k}}$ Modified from Kassam $e t$ al., 2010 to include medicinal plants used in treating laryngitis, ${ }^{\mathrm{l}}$ Medicinal plants used in treating bones and muscle, ${ }^{\mathrm{m}}$ Medicinal plants in this category require incantation, however, it is not advisable to practice it without proper consultation with the traditionalists. It is a psychic's warning as provided by the orthodox practitioners (traditionalists, diviners and herbalists among the informants). ${ }^{n}$ Medicinal plants used for the treatment of diarrhea, ${ }^{\circ}$ Medicinal plants used in treating shortage of red cells or haemoglobin in the blood.

\section{Results and Discussion}

Demographic characteristics of the Informants/participants in the group discussion.

This research study revealed that traditional healing in these communities is being practiced by both men and women. This was observed among herbal vendors (those who engage in the sales and trade of some types of medicinal plants in the market) and herbalists (those who diagnose and prescribe at home). 
Females engaged more in the sales of herbal medicine than males, which contrasted to what was obtained from the herbalists. The proportion of male herbalist was higher (87.5\%) than their female counterparts. This findings accord with the study of Shapi et al. (2009) that in some communities both men and women practice traditional medicine. The relationship between herbal vendors and herbalists is like that of pharmacists and the doctor in contemporary medicine. While the doctors diagnose the ailments and prescribe the treatment, the pharmacists are regarded as the chemists who interpret the composition of the prescription and the physical properties. After the patient has been diagnosed for an ailment by the herbalist, herbal vendors are consulted to provide information on the chemistry, composition and physical properties of medicinal plants as prescribed by the herbalist. This is a clear indication that there is a synergy in the operation of the two, based on indigenous knowledge both of them have acquired through training as apprentices in the sales of the medicinal plants or as it was handed down to them by their grandparents, especially, for the traditional practitioners. The age range of the herbalists varied between $35-78$ years while the vendors were between the age range of 28 -64 years. Children of the herbalists and the vendors constituted a greater percentage of the apprentices $(60 \%)$ and were in the age range of 16 and 25 years. Apprentices who were 40 years of age and above were very few compared to those in the lower age group. Involvement of participants' children as apprentices in these areas is a way of transferring indigenous knowledge of medicinal plant use from one generation to the other.

\section{Indigenous Knowledge of medicinal plants}

All key informants and herbal vendors expressed their opinions on how Indigenous knowledge of medicinal plants is being transferred and retained in these communities. This is being sustained through training of their children as apprentices in trading and practicing of traditional medicine. They equally reiterated their strong belief in orthodox medicine as a means of tackling ailments including those that defy human reasoning or are considered spiritual (i.e psychic's). In such cases, the herbalist can act as a diviner thereby consulting an oracle for direction. In the studied communities, 78 plant species were identified (Table 1). The family Leguminocae constitute the highest number (8), followed by the family Euphorbiaceae (6) and Meliaceae (4) while the family Amaranthacea, Annonaceae, Combretaceae, Compositae and Rubitacea have the same number (Table 1). Trees ranked highest (60\%) followed by herbs (30\%) while shrubs were the least common (10\%). Some of these plant species also served other social and economic purposes such as source of food, vitamin, body ointment, purgative, blood tonic and other spiritual purposes.

Forty ailments that were categorized into thirty one ailments were identified to have been successfully treated by traditional medicinal plant at the study communities (Tables 1 and 2). Among the ailments that have been successfully treated through application of different parts of the plants include malaria and dysentery, malaria fever being the commonest. Others include hypertension, Anemia, yellow fever, typhoid, diabetics, jaundice and wide fontanel in children. For instance, 27 species of the total plant species identified in the markets were used in the treatment of malaria (Table 1). Therefore, for every three identified plant species, one can be used in the treatment of malaria. This was followed by the 22 plant species used for the treatment of dysentery. Next to this was the plant species used for treating skin diseases, diarrhea, cough, gonorrhea, yellow fever, diabetes etc.

The number of plant species exclusively used for treating one particular category of ailment appeared to be few (Table 2). The largest number of species identified ( seven) were solely used for treating malaria followed by those used for treating dysentery medicine, dermatological aid, venereal disease with six identified plant species.Three reasons can be deduced from these findings. First, the herbal vendors interviewed "choose" to trade herbal medicine used for the treatment of malaria, the commonest disease in the area, more than those used for other purposes. Second, the herbal vendors and the traditionalists might enjoy highest patronage from patients suffering from malaria as compared to those suffering from other ailments. Third, plant species used in the treatment of malaria are readily available in these communities. Other diseases that have lowest frequency of treatment are considered not common in these communities; they include convulsion, rheumatism, arthritis, fontanel, mental disorder, syphilis, laryngitis etc. This is because one or two plant species were identified to be used for their treatment (Tables $1 \& 2$ ).

Methods of use/preparation: These medicinal plants were prepared in various forms for the various ailments depending on the uses.

\section{Mixtures Concoction and decoction}

Some of the identified plants are either prepared in form of concoction or decoction. Concoction here referred to herbal medicine that were cooked, it could be a mixture of two or more medicinal plants or it might be a species of plants while decoction referred to those that are not cooked but can be soaked in water or wine or local gin. In most cases plant such as Allium sativum served as preservatives. Supplement News (2005), reported that one of the several indigenous remedy plants employed by local traditional healers in South West Nigeria as immune booster in the palliative management of immune suppressive diseases is the cold decoction of the leaf and seed of Phyllanthus amarus . 
In this study, Phyllanthus amarus is used in the treatment of hives and small pox when the leave is grinded and mix with ointment (Ori). The use of Ocimum basilicum leave in form of decoction in the treatment of dysentery, loss of appetite, sleeplessness is equally observed and documented in this study. This is in variance with the findings of Ilori et al., (1996) as reported in South East Nigeria, the authors opined that Ocimum spp is commonly available as spice in local savoury dishes and have been used in the treatment of different diseases such as upper respiratory infections, diarrhea, headaches, fever, ophthalmic, skin diseases and pneumonia.

\section{Mixtures of the plants' parts with ointment (Ori) or soap (Ose-dudu) as body lotion or bathing soap}

This includes plants that are grounded and mixed with ointments as body lotion or mixed with soap for bathing. Plants in this category includes the leaves of Chasmanthera dependis mixed with body ointment for the treatment of fracture, swollen leg or wound. The leave of Chenopodium ambrasioides mixed with body ointment in treating arthritis. Others include mixture of Euphorbia laterifolia with palm oil or body ointment; mixture of Peperomiape lucida leave with body ointment for treating small pox; mixture of Phyllanthus amarus with ointment for treating hives; Tetrapleura tetraptera are grinded together and mix with local soap (Osedudu) for bathing in treating skin disease. In most cases the ointments referred to are locally produced oil extracts from Vitellaria paradoxa known as "Ori" and the bathing soap is locally produced from ash known as "Ose-dudu". Current findings are similar to Olowokudeejo et al., (1993) who reported that medicinal plants are prepared in the form of powder, decoction, bathing soap, soup and the majority of them are used in mixtures of or concoctions of materials while a few are used as single.

\section{Mixtures of the plants' parts with other plant species}

Combination of some of the identified plant species such as Khaya spp, Ageratum conyzoides either in the form of concoction or decoction in the treatment of Malaria (a prevalent ailments in the study communities) was documented. This support the findings of Fabeku and Akinsulure, 2008 , that combination of the bark of Khaya spp (Epo Oganwo), bark of Alstonia boonei (Epo ahun), fruits of Xylopia aethiopica (Eleru-Alamo) soak with water are used in the treatment of malaria in Nigeria. The research finding also revealed that the root of Nauclea latifolia soak in water with Eugenia aromatica are used in the treatment of Malaria in these communities. The leave of Morinda lucida mix together with the bark of Khaya spp are used in the treatment of Malaria are equally reported. This is in tandem with Sofowora, (1993) and Odugbemi et al., 2007 that Morinda lucida and Khaya spp were found to be in use for malaria therapy in Okeigbo area of Ondo-State, Nigeria. The mixtures of other plant species in treating ailments such as Anemia, dysentery and diabetics were also observed as presented in Table 1.

\section{Other methods of use}

Other methods of use include chewing stick, soup condiment and grinding into powdery form. The fruits of Lagenaria breviflorus can be thrown on the floor around the houses while the bark of Erythrophellum suaveolens can be soaked in water to scare away evil spirit. There are also those reported to be used as gourd to keep medicine. This involves the use of various parts like bark, stems, leaves, seeds, pods, bulbs, nuts, roots and fruits. For instance, inflammation treatments can best be administered in the form of cream or pomade while teething problem or mouth disease can best be treated by chewing the stick or the stem of the plant and sometimes the patient might prefer herb preparation in the form of a concoction to a decoction. The barks of species of plant like Acacia nilotica (Booni) can be used in combination as a burnt incense to scare away or repel evil spirit or in the form of decoction to treat dysentery. The use of herbal decoction, extracts of medicinal plants and their various parts for treatment of ailments have been reported by several researchers (FAO 1983; Gbile et.al 1985; Anwana and Obot 2003).

\section{Plants use by frequency of use and preparation methods}

All the species of plants used in the treatment of common ailments such as malaria, diarrhea, dysentery, yellow fever, jaundice, hypertension, diabetic e.t.c can be considered as the frequent. This includes 27 plant species used in the treatments of malaria, 21 species used for the treatment of dysentery e.t.c. 14 species of the identified plants are used in the treatment of up to three to four ailments either in the form of concoction or decoction. For instance, Xylopia aethopia can be used as concoction or decoction for the treatment of Skin disease, purgative, diabetes and dysentery, Vitellaria paradoxa can be used in the treatment of malaria, hypertension and also in the production of body ointment, and as a vitamin supplement; depending on the parts of plants used and their methods of production. Parkia biglobosa can be used for the treatment of malaria, intestinal incontinence and mental disorder; also as tonic. Also the root of Aristolochia ringens can be used in the form of decoction either soaked in water or local gin in the treatment of typhoid or malaria. Kubmarawa et al., (2007) noted that Aristolochia albida and Nuclea diderichii are reported to be used as antimalarial in Riji, Adamawa State, North eastern Nigeria. 
Chasmanthera dependeus was found useful in the treatment of four ailments including fracture, swollen leg, wound and for children with weak bones; this is in the form of decoction. Among these multipurpose medicinal plants, trees constituted the highest percentage (about 65\%) of the identified medicinal plants, followed by herbaceous plants (about 32\%), and shrubs were the least (about 13\%).

\section{Patient patronage and Local Knowledge}

The vendors and the traditionalists gave various reasons for having different levels of clientele, the most important of which includes the affordable price of the herbal medicine. For instance, the cost of preparing, concoction or decoction for the treatment of malaria or dysentery ( $\$ 1$ and $\$ 2$ per dosage) does not have a standard or precise measurement. Measurement is based on the discretion of the user and his/her level of response to treatment. Traditionalists and some herbal vendors also offer spiritual consultation (especially for ailments considered as psychic) to their patients on matters such as provision of repellant of evil spirit, causes of infertility, broken relationships, loss of patronage or businesses success and causes of delayed in marriages. In this case, the herbalists can decide to act as diviners when sacrifices are offered and oracles are consulted. They also treat ailments where the cost of contemporary medicine is considered too exorbitant. Another reason given for the increased level of patronage being enjoyed by the herbal vendors includes bottleneck and bureaucratic procedures; associated with contemporary medical consultant and nurses in public hospitals as compared to the easily accessible herbal vendors and traditionalists. All the reasons provided support the findings of Kassam et al. (2010) that heavy reliance on plant medicine or self-treatment may be the major option and local knowledge of medicinal plants might save lives due to their relative accessibility, low prices, general acceptance by local communities and the inadequacy of health centers especially in rural areas. The challenges occasionally faced by patients include long queues at the points of collection of patient cards and at the places that are set up for meeting the doctors. Poverty could be the underlining factor that many patients find it difficult to patronize privately owned hospitals due to high charges, although their services are relatively more efficient.

\section{Therapeutic rationale behind formulation}

The rationale behind formulation of these herbal mixtures or single dose can be attributed to believe and trust in curative remedies of these plant species. There are indications that adherence to these traditional believes have been sustained through a formidable and organized indigenous knowledge system handed from one generation to the other as reported by the herbal vendors and the herbalist. Elujoba, (2005) emphasized that it is believed very strongly that if the herbs never worked for our predecessors, malaria would have devastated Africa and the missionaries that came to scramble for Africa would not have met a single person on the continent of Africa. World Health Organization International Agency for Research on Cancer (WHO-IARC, 2002), opined that due to the complexity of herbal and botanical preparations, information on the active principles of plant derived products is incomplete or unavailable this explains reason why the therapeutic rationale behind their formulation is lacking. The author therefore concluded that it is a function of the traditionally -held belief that the synergistic combination of several active principles in some herbal preparations is responsible for their beneficial effects.

Apart from believe there is no therapeutic rationale behind the usage and formulation of these herbal medicines in these communities hence a scientific study needed to be initiated. This forms part of the view of a group of experts invited by the World Health organization Regional Office for the Western Pacific that most herbal medicines still need to be studied scientifically, although the experience obtained from their traditional use over the years should not be ignored (WHO, 1993). The group went further to illuminate on the fact that since there is not enough evidence produced by common scientific approaches to answer questions of safety and efficacy about most of the herbal medicines now in use, the rational use and further development of herbal medicines will be supported by further appropriate scientific studies of these products, and thus the development of criteria for such studies.

\section{Dosage and Unit of measurement}

One of the challenges encountered in this study was on the question of unit of measurement used in the administration of these drugs. This is because no standard measurement for dosage was presented even upon request by the researchers. This is due to lack of standard of measurement for the dosage. The data obtained shows that there is observable difference in the quantity of dosage delivered by vendors. For instance, in a situation where a cup of the concoction was prescribed to be taken in the morning or afternoon or night by one vendor while the other vendor may prescribe larger/smaller volume of the same medicine for the same ailment. Therefore, the greater challenge that traditional medicine is facing is the lack of unified or standardized unit of measurement. For instance, cases of patients who mistakenly assumed a cup of dosage to be equivalent to $500 \mathrm{ml}$-cup rather than $250 \mathrm{ml}$ have been recorded as severe. This is unlike contemporary medicine, where dosage is stipulated and in most cases unit of measurement is found attached to the prescribed drug. A cup of dosage as prescribed by the vendors or traditionalist could be assumed to mean either a tumbler of 50ml or a stainless or 
mug cup of $300 \mathrm{ml}$. The decision to use either a tumbler of $50 \mathrm{ml}$ or a stainless or mug cup of $300 \mathrm{ml}$ as unit of measurement as prescribed by the herbal vendors or herbalist is at the discretion of the patient. The patient therefore rely on the information obtained on the method of preparation and the concentration of the content. If the herb is prepared and soak in a local gin there is every tendency that the concentration will be very high hence a tumbler cup of 50ml represent the unit of measurement as a cup. On the other hand, if the herbal medicine is prepared with water, a stainless or mug cup of $300 \mathrm{ml}$ is preferably considered. Other units of measurement commonly used as identified in this study include a tablespoon. A tablespoon could vary between $5 \mathrm{ml}$ and $10 \mathrm{ml}$ depending on the size. Thus, confusion may arise in the use of this unit of measurement.

\section{Other socio-economic uses of medicinal plants}

The study revealed that in addition to their medicinal values, some of the identified plants offer other socioeconomic benefits. For instance, the oil extracts from the seed of Vitellaria paradoxa, apart from serving as ointment can also serve as vegetable oil and is used for cooking and in remote rural areas it could serve as biofuel for the locally produced lamp. The seeds of Parkia biglobosa can also be processed to serve as soup condiment locally called "iru". Medicinal plants are grouped for many commercial purposes in the broader category "medicinal and aromatic plants" (MAPs), covering plants used not only medicinally (Sensustricto), but also for neighbouring and overlapping purposes, for instance, as foods, condiments and cosmetics (Hamilton 2003). The research finding also revealed that fruit of Mangifera indica can be processed at a commercial scale for the production of juice, while the seeds of Capsicum frutescens can be used as a stimulant. All the key informants and participants in the group discussions were familiar with the use of these plant species for various purposes as indicated. This is largely because apart from their medicinal values these plant species constituted greater proportion of their food. Similar research studies have been undertaken on the social and economic values of some of these identified plant species as reported in literatures. For instance, the mango, Mangifera indica is well known for its excellent exotic flavor and commercial production has been reported in more than 87 countries (Sivakumar Jiang et al., 2010). In addition to its use as a stimulant, medicinal values of Capsicum frutescens was observed among the locals in Weno, Romanum, and Pilis islands, Chuk Atoll, Federated States of Micronesia (Yamamoto., 2013). The researcher reported that when asked about medicinal uses of Capsicum, three people responded that the peppers are used to treat toothaches. In other regions of Oceania, the fruits, seeds, and leaves are used to treat various ailments such as painful joints, back pain etc.

\section{Method of collection or source}

We found that it was the norm for traditionalists to maintain home botanical gardens to meet their immediate medicinal needs rather than visiting markets to purchase. All the herbaceous plants identified can be regarded as being successfully domesticated and they can be found grown at the back yard or on the cultivated farm unlike trees. Tree species such as Kigellia africana, Annona senegalensis, Tetraptera teraptera used for the treatment of ailments such as malaria, dysentery and rheumatism can only be obtained in the wild. It can therefore be deduced that the host communities have recorded success in domesticating and cultivating the herbaceous and shrub medicinal plant species compared to the trees. Domestication of indigenous plants have been emphasized as one of the ways of achieving ex-situ conservation of various species of plants and animals of threatened or endangered species (Nigeria First National Biodiversity Report 2001). Aframonum melegueta, Allium ascalonicum, Allium sutivum, Erythrococca anomala, Eugenia aromatica, Garcinia kola and Xylophia aethiopica were said to be non -native but were sought or bought from other places outside these communities.

\section{Conclusion}

The selected communities adjoining Old Oyo National Park have an intricate link between indigenous knowledge and medicinal plant use. This study has established that the traditionalists (herbal vendors and herbalists) possessed local knowledge of medicinal plants use which they transfer or pass from one generation to the other by involving their biological children and other young ones (non-biological children ) in herbal sales and traditional healing. This is a clear demonstration of how indigenous knowledge of medicinal plant use is being retained and transferred from one generation to the other.

Various plant species were identified to treat different ailments, in addition to using the same species as sources of food and raw materials, among others. Seventy-eight plant species were identified to be used for the treatment of forty ailments in thirty-one categories across the communities. Trees ranked highest (60\%) followed by herbs (30\%) while shrubs were the least common (10\%). Some of these plant species also served other social and economic purposes such as sources of food, vitamins, body ointment, purgative, blood tonic and other spiritual purposes. 
Forty ailments that were categorized into thirty one were identified to have been successfully treated by traditional medicinal plant at the study communities. The names of some of the identified ailments that have been successfully treated through application of different parts of the plants include malaria and dysentery. Others include hypertension, Anemia, yellow fever, typhoid, diabetes, jaundice and wide fontanel among the children. These medicinal plants were prepared in various forms for the different ailments according to their uses. There are those used as concoction, decoction or as both. This involves the use of various parts as bark, stem, leaves, seed, pod, bulb, nut, root and fruits.

It can also be deduced that the host communities have recorded success in domesticating and cultivating the herbaceous and shrub medicinal plant species compared to the trees. This is because tree species such as Kigellia africana, Annona senegalensis, Tetraptera teraptera used for the treatment of ailments such as malaria, dysentery, rheumatism can only be obtained in the wild. Species of plants such as Aframonum melegueta,Allium ascalonicum, Allium sutivum, Erythrococca anomala, Eugenia aromatica, Garcinia kola and Xylophia aethiopica were said to be non -native and they were sought or bought from other places outside these communities.

The vendors and the traditionalists gave various reasons for having different level of customer's patronage and the most important include the affordable price of the herbal medicine. One of the challenges faced in this study was the question of unit of measurement used in the administration of these drugs by the herbal vendors or sellers and the traditionalists or the herbalists. This is due to the lack of standard of a measurement for the dosage.

Capturing local knowledge on medicinal plant use is recognized today as the most viable method of identifying new medicinal plants or refocusing on those earlier reported for bioactive constituents. The study will contribute to the advancement of contemporary medicine, resource efficiency and development of new enterprise in the selected communities.

\section{Recommendations}

Based on the findings of this study, there is need to create a data base of information on medicinal plant species used by the study communities including their methods of application for various ailments. Further research works also need to be carried out on the laboratory analysis of these species to identify the active ingredients as a way of synthesizing some of these plants for the production of drugs used in modern day medicine. The therapeutic or prophylactic indications of the identified herbal medicines can only be established through thorough and in-depth scientific research. Comprehensive quantitative study on the social and economic importance of the medicinal plants is recommended. Issues related to standardizing dosage also need to be addressed as a way of saving life, resource and time. Methods of domesticating the identified medicinal plant species, especially the trees mostly being collected from the wild need further research. Policy and development are also lacking and they need to be included in the research.

\section{Acknowledgement}

Financial support of the management of Nigeria National Park Service is hereby acknowledged. Also, Old Oyo National Park supported this project through assigning the staff that accompanied the researchers during the course of this study to facilitate interaction with the host communities.

Conflict of Interest: The authors whose names are listed below hereby declared that there is no conflict or affiliations or involvement in any organization or entity with any financial or non-financial interest on the submitted manuscript titled: Capturing Indigenous Knowledge on Medicinal Plants Use: Case study of selected communities in Old Oyo National Park, Nigeria.

\section{References}

1. Agbaje-Williams, B.(1983).A contribution to the Archeology of Old Oyo. PhD Thesis, Department of Archeology, University of Ibadan, Nigeria.

2. Akinjogbin, I. A. (1966). A Chronology of Yoruba History 1789-1840. Odu, 11(2), 81-86

3. Alarape, A. (2002).Culture and Conservation in and around Old Oyo National Park. PhD Thesis Submitted to the Department of Wildlife and Fisheries, University of Ibadan.

4. Anwana E.D and Obot. E. A (2003). Ethnobotany of Human Settlements in Cross -River National Park, Okwango Division Useful and Medicinal Plants. ROAN. Journal of Conservation Vol.1.No1 \&2, 34-46

5. Arbonnier, M. (2004). Trees, shrubs and lianas of West African dry zones. ISBN Margraf Publishers, GMBH

6. Baishya, R .A., and Begum, A. (2013). Promotion of Rural livelihood through Medicinal and Aromati Plants based Cottage Industries for upliftment of rural economy of Assam Rat. Open Access Scientific report. 2(1):1-4 [Online]URL: http://dx.doi.org/10.4172/scientificreports.619 
7. Betti, J. (2004). An Ethnobotanical Study of medicinal plants among the Baka Pygmies in the DJA Biosphere reserve, Cameroon. African Study Monograph 25(1):1-27

8. Ceballous-Lascurain. (2001). Integrating Biodiversity into the Tourism Sector: Best Practice Guidelines a report delivered to the United Nations Environment Programme, the United Nations Development Programme, the Global Environment Facility and the Biodiversity Planning Support Progamme in June 2001.

9. Charter, J.R (1970). Vegetation Ecological Zones. Unpublished M. Sc Federal Department of Forestry, Ibadan

10. Choudhary, B. (2002). The new international seed treaty: Promises and prospects for food security. Current Science 83: 366-369

11. Elujoba, T (2005): "Traditional Medicinal Plants and Malaria (Book Review)". Africa Journal of Traditional .CAM, 2(2): 206-207

12. Fabeku, P. O and Akinsulire, O (2008): The role of traditional medicine among the Yoruba of South-West Nigeria. In: Odugbemi Tolu (ed.) Medicinal plants from Nigeria. University of Lagos Press, Nigeria. Pp 43-54

13. Food and Agricultural Organisation (1983). The importance of medicinal forest plants in Africa

14. and Latin America .F.A.O Forestry paper 21.Pp 16-17

15. Gbile, Z.O., Soladoye, M.O and Adesina. A. K (1985). Plants in the traditional medicine in West Africa In: Modern studies in African Botany. (eds. Goldblast P.P and Lowey, P.P) Missouri Botanical Garden, Missouri, U.S.A, pp 343-34

16. Geerling, C. (1973). Vegetation of Upper Ogun and Old Oyo Game reserve, Department of Forestry, University of Ibadan.

17. Hamilton, A. (2003). Medicinal Plants and conservation: Issues and Approaches. International Plants Conservation Unit, WWF-UK, Survey, UK Nigeria First Biodiversity Report. 2001

18. Ilori, M.A.O, Sheteolu, E.A, Omonigbehin and A. A Adeneye (1996) 'Antidiarrhoeal activities of Ocinum gratissimum'. Journal of Diarrhoeal Diseases Research 14:283-285

19. Kassam K. A, Munira Karamkhudoeva, Morgan Ruelle and MichelleBaumflek (2010): Medicinal Plant Use and Health Sovereignty: Findings from the Tajik and Afghan Pamirs. Human Ecology. 38:817-829.

20. Kayode , J (2006).Conservation of Indigenous medicinal botanicals in Ekiti-State, Nigeria. Journal of

21. Zhejiang University SCIENCE -B 7 (9):713-718

22. Keay, R.W.J., (1959). An outline of Nigerian vegetation. Government Printer, Lagos

23. Keay, R.W.J., Onochie, C.F.A. \& Stanfield, D.P. (1964). Nigerian Trees. Federal Department of Forest Research, Ibadan, Nigeria Published by the Department of Forest Research, Ibadan, Nigeria. pp. 1-40.

24. Kubmarawa, D.A.G, Ajoku, N.M, Enwerem and D.A Okorie (2007). 'Preliminary Phytochemical and Antimicrobial Screening of 50 Medicinal plants from Nigeria ". Africa Journal of Biotechnology: 6(14): 1690-1696

25. Matsheta, M.S., and Mulaudzi, F.M. (2008). The Perceptions of Traditional Healers of Cervical Cancer Care at GaMothapo Village in Limpopo Province. Indilinga: African Journal of Indigenous Knowledge Systems, 7:103-116.

26. Moerman, D. E. (1998). Native American Ethnobotany. Timber Press, Portland, Oregon.

27. Odugbemi, T. O, O.R. Akinsulure, I.E. Aibinu, P.O. Fabeku (2007). "Medicinal plants useful for Malaria Therapy in Okeigbo". African Journal of Traditional, Complementary and Alternative Medicine Vol. 4. No. 2 Pp 191-198

28. Odugbemi, T. (2008).Outlines and pictures of Medicinal Plants from Nigeria. University of Lagos Press Lagos, Nigeria

29. Oladeji, S.O and Adeyemo, A. I (2012): Ecological distribution of floral resources of Medicinal values in Old Oyo National Park, Oyo-State,. Nigeria 4th Conference of Forest and Forest Products Society of Nigeria, Federal University of Technology, Akure, Nigeria, $16^{\text {th }}-18^{\text {th }}$ March, 2012, Adekunle, V., Onyekwelu, J and Oke, D.O (eds), 150-166

30. Oladeji, S.O and Agbelusi (2014): Conservation status of fauna and flora resources composition and distribution in Old Oyo National Park: A key for sustainable natural resource management. Journal of Environment and Ecology Management. 2014(4): 1-30. [Online] URL: http://scik.org

31. Olowokudeejo, J.D., Adeloye .O.A.\&Fadeyi . M.O.1993. Comparative Phytochemistry and Anatomy of Jatropha (Euphorbiaceae) species used in Herbal Medicine in Nigeria. Journal of Scientific Research and Development 1:20-23.

32. Owuor, B., and Kisangau, D. (2006): Kenyan medicinal plants used as antivenin: a comparison of plant usage. Journal of Ethnobiology and Ethnomedicine. 2(7):1-8 [online] URL :http://www.ethnomed.com/content/2/1/7

33. Owuor, B.O.,Mulemi,B.E., and Kokwaro,J.O (2005): Indigenous snake bite remedies of the Luo of Western Kenya Journal of Ethnobiology 20:129-141

34. Pesek T.J., Helton L.R., and Nair, M (2006): Healing across Cultures: Learning from traditions. EcoHealth 3(2): 114-118

35. Posey, D.A. (1988).The declaration of Beleem.In: Proccedings of the $1^{\text {st }}$ International Congress of Ethnobiology. Posey, D.A and W. Overal Ed. Muse ParaenseGoeldiBeelem.

36. Posey, D.A. (1999).Cultural and spiritual values of biodiversity.A complementary contribution to the global biodiversity assessment in cultural and spiritual values of biodiversity.Posey D.A. Ed. 1-9, UNEP and Intermediate Tech. Publications, London.

37. PROTABASE LINK: http://database.prota.org/serach.htm, http://www.prota.org/PROTA Start frames.htm accessed $12 / 02 / 2017$

38. Ramakrishnappa, K. (2002). Impact of Cultivation and Gathering of Medicinal Plants on Biodiversity Case studies from India. In: Biodiversity and Ecosystem. Approach in Agriculture, Forestry and Fisheries (online) FAO, 2002.

39. Shapi M., Matengu, K., and Mu Ashekele, H (2009). Indigenous Knowledge System Pilot Study Oshikot Region. Multidisciplinary Research Centre, University of Namibia, Windhoek.

135 
40. Sivakumar, D., Jiang, Y., and Yahia, E, M. (2010): Maintaining mango (Mangifera indica L.) fruit quality during the export chain. Food Research International 44 (2011): 1254-1263

41. Sofowora, A. (1993). Medicinal plants and Traditional Medicine in Africa . Published by Spectrum Books Ltd., Ibadan

42. Srivastava, R. (2000). Studying the information needs of medicinal plant stakeholders in Europe. Traffic Dispatches, 15(5), 13.

43. Subramanyam., R, Newmaster.,C,S, Murugesan., M, Balasubranmaniam.,V and Muneer., M,U.(2008).Consensus of the 'Malasars'traditional aboriginal knowledge of medicinal plants in the Velliangiri holy hills,India. Journal of Ethnobiology and Ethnomedicine 4(8):1-14BioMed Central Ltd Online].URL.http://www.rthnobiomed.com/content/4/1/8

44. United Nation Education Science and Cultural Organisation (1996). unesdoc.unesco.org/images/0016/001631/163168e.pdf - assessed 07 January, 2013.

45. Uprety, Y., Asselin, H., Boon, E.K., Yadav, S. and Shrestha, K.K (2010). Indigenous use and bio-efficacy of medicinal plants in the Rasuwa District, Central Nepal. Journal of Ethnobiology and Ethnomedicine : 6:3

46. Wakdikar., S.(2004). Global health care challenge: Indian experiences and new prescriptions. Electronic Journal of Biotechnology, North America, 715122004

47. World Health Organisation. (1980). Global Demand for Medicinal and Romantic Plants.

48. WHO (1993) Research Guidelines for Evaluating the Safety and Efficacy of Herbal Medicines, Manila

49. World Health Organization International Agency for Research on Cancer (WHO-IARC, (2002): Some traditional Herbal medicines, some mycotoxins, Naphthalene and Styrene. Vol. 82 IARC Press, Lyon France

50. Yamamoto, S. (2013): Use of Capsicum frutescence in Weno, Romanum and Piis islands, Chuk Atoll, Federated States of Mironesia. Occasional Papers 53(2013): 77-89.

51. Yakubu, F.B and Idumah, F.O (2002). Processing and Marketing of Charcoal in Selected Local Government Areas of OyoState, Nigeria. In: Forestry and challenges of sustainable livelihood. Proceedings of the 28th Annual Conference of the Forestry Association of Nigeria, Akure, Ondo-State. Ed.Abu, J.E, Oni , P.I and Popoola, L. 